\title{
Evolution of fracture normal stiffness due to pressure dissolution and precipitation
}

\author{
Philipp S. Lang ${ }^{1}$, Adriana Paluszny, Robert W. Zimmerman \\ Deparment of Earth Science and Engineering \\ Imperial College, London SW7 2AZ, UK
}

\begin{abstract}
The normal stiffness of a fracture is a key parameter that controls, for example, rock mass deformability, the change in hydraulic transmissivity due to stress changes, and the speed and attenuation of seismic waves that travel across the fracture. Non-linearity of normal stiffness as a function of stress is often attributed to plastic yield at discrete contacts. Similar surface-altering mechanisms occur due to pressure solution and precipitation over larger timescales. These processes partition the fracture surfaces into a flattened contact region, and a rough free surface that bounds the void space. Under low loads, contact occurs exclusively over the flattened part, leading to rapid, exponential stiffening. At higher loads, contact occurs over the rough surface fraction, leading to the conventional linear increase of stiffness with stress. It follows that a relationship exists between the history of in situ temperature and stress state of a rock fracture, and its subsequent deformation behavior.

Keywords: Rock fracture; Stiffness; Compliance; Diagenetic processes;

Pressure solution; Precipitation
\end{abstract}

\section{Introduction}

Fractures are widespread in the Earth's crust and often control the overall behavior of rock masses at large scales. Heat and mass transport, fluid flow, and

\footnotetext{
${ }^{1}$ Corresponding author: p.lang13@imperial.ac.uk
} 
the velocity and attenuation of seismic waves are all generally stress-dependent in fractured rock. Normal stiffness is a key parameter that relates relevant fracture properties to the magnitude of the normal stress. As such, it represents the stiffness contribution attributable to the closure of the void space between the rock walls, and the excess compression of contacting asperities therein with respect to the intact rock (Cook, 1992). The fracture normal stiffness is the main factor that controls the P-wave transmission and reflection coefficients (PyrakNolte et al., 1990), and is closely correlated to the hydraulic transmissivity (Pyrak-Nolte and Morris, 2000).

The numerical value of the normal stiffness will strongly depend on the morphological properties of the fracture, such as roughness and the correlation between the surfaces in contact, and the elastic moduli of the rock (Zimmerman et al., 1990; Hopkins et al., 1990; Pyrak-Nolte and Morris, 2000). Chemicallymediated processes may significantly alter fracture morphology, thereby changing the hydro-mechanical properties of a rock fracture after formation (Chester and Logan, 1986; Moore et al., 1994; Polak et al., 2003; Laubach et al., 2004; Yasuhara et al., 2006). These processes occur either as diagenetic mechanisms over geologic timescales at in situ conditions, or during engineering procedures, where injected fluids and induced temperature changes can accelerate these processes significantly. In open fractures, where opposing surfaces are not in nominal contact, the process of precipitation dominates, to structurally seal the discontinuity over time (Laubach, 2003). The formation of bridging structures precedes this sealing (Lander and Laubach, 2015), and these structures control the deformation behavior of the fractures (Sayers et al., 2009). Large fractures that form highly connected networks are expected to be under compression at appreciable depth (Barton et al., 1995; Zoback, 2007). The relative displacement between the opposing rough rock surfaces, and the resulting partial contact, give rise to their effect on fluid flow, transport and displacement (Zimmerman et al., 1990, 1992; Borri-Brunetto et al., 1999). For these fractures, the coupled processes of pressure solution and free-face precipitation constitute significant diagenetic mechanisms (Gamond, 1987; Gratier and Gamond, 1990; 
Peacock and Sanderson, 1995). Their combined effect leads to a redistribution of material, driven by spatial gradients in chemical potential, from the mechanically stressed grain contacts to the mechanically open pore space (Weyl, 1959; Rutter, 1976; Gratz, 1991; Lehner and Leroy, 2004). Although the hydraulic response to such compaction processes on fractures have been extensively studied (Moore et al., 1994; Durham et al., 2001; Morrow et al., 2001; Polak et al., 2003; Yasuhara et al., 2006), quantitative studies of the mechanical effects are sparse, and they remain experimentally challenging.

Building on previous work (Lang et al., 2015), the present study presents coupled hydro-mechanical-chemical simulations at the pore scale to assess changes in fracture normal stiffness under the effects of pressure solution and precipitation, for a water-quartz system. Specifically, instantaneous fracture closure curves are generated, for specific points in time during the dissolution and precipitation process, and related to changes in the rock surface morphology (Figure 1). For a fracture undergoing pressure dissolution, the area of discrete contact between the two rough surfaces increases, the number of contact patches that make up this discrete contact area increases, and the dominating contact regime changes from dissolved, smoothened contacts to unchanged, rough contacts. The ensuing changes in fracture closure behavior are in qualitative and quantitative agreement with experimentally obtained curves for multiple compression cycles on unmated fractures, where plastic damage leads to similar alterations of the rock surface morphology.

\section{Methodology}

The normal stiffness of a fracture is largely attributable to the frictionless contact between two rough surfaces. This contact problem is equivalent to the contact between a flat, elastic body with composite moduli, and a rigid body with a composite profile (Brown and Scholz, 1985). The profile of the composite surface is obtained as the sum of the opposing surface heights, and thus represents the aperture profile at unstressed conditions, i.e., when the two rough 


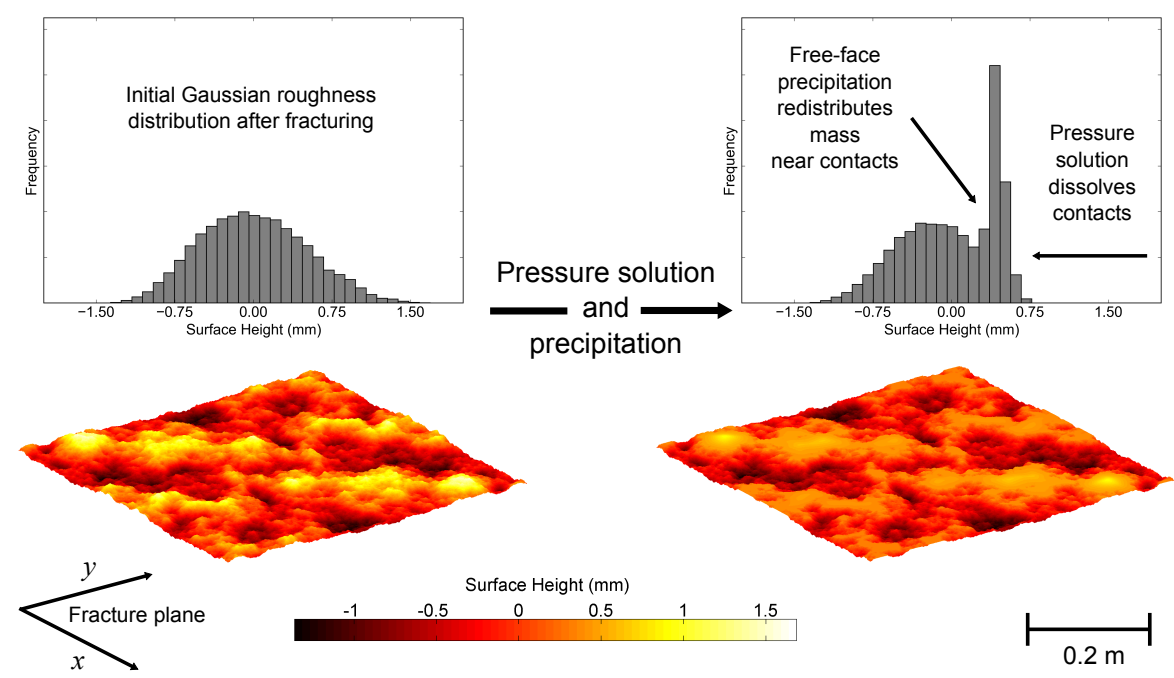

Figure 1: A $0.5 \times 0.5 \mathrm{~m}$ periodic fracture composite surface in its initial state (left) and after pressure solution and free-face precipitation acted for $300 \mathrm{ka}$ at $10 \mathrm{MPa}$ effective confining pressure and $150{ }^{\circ} \mathrm{C}$ (right).

surfaces touch at a single point. A fracturing process produces two surfaces of isotropic, self-affine nature of approximately Gaussian height distribution (Brown and Scholz, 1985; Poon et al., 1992; Schmittbuhl et al., 1995). The roughness power spectrum $C(q)$ sufficiently characterizes both the root-meansquared roughness, and the height correlation of such surfaces as (Nayak, 1971):

$$
C(q)=\frac{1}{(2 \pi)^{2}} \int\langle h(\boldsymbol{x}) h(\boldsymbol{o})\rangle \mathrm{e}^{-\mathrm{i} \boldsymbol{q} \cdot \boldsymbol{x}} \mathrm{d}^{2} x
$$

where $\boldsymbol{q}$ is the roughness wave vector, and $q=|\boldsymbol{q}|$ is its magnitude, the wavenumber, or spatial frequency. For measured data of $h(\boldsymbol{x})$, which are usually shifted to obtain $\langle h\rangle=0$, Equation 1 can be evaluated using a Discrete Fourier Transform and radial averaging. A numerical algorithm based on a mathematical recipe in Persson et al. (2005), pp.76-79, has been implemented for this purpose. An ideal spectrum $C(q)$ for surfaces of this kind is given by (e.g. Pastewka et al., 
2013)

$$
C(q) \propto \begin{cases}1 & q_{L}<q<q_{0} \\ q^{-2(H+1)} & q_{0} \leq q \leq q_{1} \\ 0 & q_{1}<q<q_{\ell}\end{cases}
$$

In Equation 2, $q_{L}=2 \pi / L$ is the smallest roughness frequency that can possibly occur in the surface, limited by the sample length $L$. This wavenumber $q_{L}$ corresponds to the largest possible roughness wavelength $\lambda_{L}=L / 2 \pi$ occurring in the surface. At the other end of the spectrum, the largest possible roughness frequency, $q_{\ell}$, or smallest roughness wavelength, is limited by the lattice size $\ell$. Note that $q_{L}$, or $\lambda_{L}$, is a consequence of the physical size of the surface of interest, and $q_{\ell}$ is a consequence of discretization. The discretization limit may result from a numerical lattice or grid size, or may result from limitations in measurement resolution. For example, a surface of length $L$ has a cell size of $\ell=L / \mathcal{L}$, where $\mathcal{L}$ is the number of equally sized square lattices. Surfaces in nature have no notion of a discretization-induced limit $\lambda_{\ell}$, but are rough down to the molecular scale (Yang et al., 2006; Luan and Robbins, 2005). A surface with roughness wavelengths bounded by its size and discretization limit is shown in Figure 2a, and the associated power spectrum in Figure 2a. Surfaces of this kind are good models of naturally induced tensile fracture surfaces (Persson, 2014), apart from having a lower limit, $q_{\ell}$, that is an unavoidable numerical artifact. In between the limits $\left(q_{L}, q_{\ell}\right)$, two more characteristic wavelengths may exist.

If the smallest roughness wavelength present on a surface is larger than the discretization limit $\lambda_{\ell}$, a so-called cut-off wavelength $\lambda_{1}>\lambda_{\ell}$ exists, and a corresponding cut-off wavenumber $q_{1}<q_{\ell}$; see Figures 2c and 2d. As a consequence, surfaces with a cut-off lack smaller roughness features; compare Figures 2a where $q_{1}=q_{\ell}$ and $2 \mathrm{c}$ where $q_{1}<q_{\ell}$. In this case, only larger roughness features are represented, i.e., the surface appears smoother for the lack of small-scale roughness. This is equivalent to a low-pass filter, in terms of roughness frequency. Although real surfaces don't feature such a cut-off, it has been shown that ignoring theses small roughness features has negligible 
effects when numerically computing normal stiffness (Campañá et al., 2011) or transmissivity (Zimmerman and Bodvarsson, 1996; Vallet et al., 2009; Neuville et al., 2011). In other words, many phenomena in fractures depend mostly on the largest roughness wavelengths of the contacting surfaces. Introducing a cut-off wavelength is (1) unavoidable when representing a surface discretely, because at the most the discretization bound represents the artificial limit $q_{1}=q_{\ell}$, and (2) a convenient means of obtaining first-order solutions with limited computational effort.

If the largest roughness wavelength present on a surface is less than the physical limit $\lambda_{L}$, a so-called roll-off wavelength $\lambda_{0}<\lambda_{L}$ exists, along with a corresponding roll-off wavenumber $q_{0}>q_{L}$. This is equivalent to a high-pass filter in terms of roughness spatial frequencies. An example of such a surface is shown in Figure 2e, and its spectrum in Figure 2f. Compared to Figure 2a, no roughness wavelength that spans the entire surface can be identified. Surfaces of this kind may represent manufactured, flat surfaces, which are only rough below a certain length-scale. A classic example is sandpaper, where the roll-off is proportional to the grind number. Such surfaces may be indicative of the aperture field formed by two correlated surfaces. A surface with both a cut-off and a roll-off in roughness is illustrated in Figure $2 \mathrm{~g}$, and its spectrum is shown in Figure 2h.

A universal scaling exponent of $H \approx 0.8$ has been found to apply for surfaces of tensile fractures across a range of different rock types and grain size distributions (e.g. Poon et al., 1992). The root-mean-square roughness of the surface controls the amplitude of the power spectrum, $h_{\mathrm{rms}}=\left\langle h^{2}\right\rangle^{1 / 2}=$ $\left(2 \pi \int q C(q) \mathrm{d} q\right)^{1 / 2}$; its length-scaling is less clear (Schmittbuhl et al., 1995; Fardin et al., 2001, 2004; Tatone and Grasselli, 2013), mostly due to the unavailability of large, undamaged tensile fracture surfaces. Surfaces formed by fracture propagation are in general expected to be fractal-like across all length scales (e.g. Persson et al., 2005), i.e., $q_{0}=q_{L}$ and $L / \lambda_{0}=1$. In fact, no evidence of a roll-off wavelength has been found for measurements over multiple length scales (Power et al., 1987; Candela et al., 2012), suggesting the exis- 
tence of roughness across all relevant length-scales in rock fractures, and, as a consequence, no obvious characteristic or homogenization length scale. Given a roughness spectrum $C(q)$, self-affine surfaces can be generated numerically through a Fourier series (Persson et al., 2005) fully periodic (Figure 1) or with periodic boundary conditions (Figure 2). The numerical accuracy of the independent algorithms for surface characterization and generation is illustrated by means of the reference and measured power spectra in Figure 2 for surfaces of $\mathcal{L} \times \mathcal{L}=1024 \times 1024$.

The observation that natural surfaces are characterized by roughness across all length scales, and that they are geometrically similar for different materials (see also Persson, 2014), has led the modeling of normal contact between two rough surfaces away from the notion of asperities, or grains (Greenwood and Williamson, 1966; Swan, 1983; Brown and Scholz, 1985; Hopkins et al., 1990), towards a self-affine roughness representation (Mourzenko et al., 1997; Persson, 2001; Hyun et al., 2004; Akarapu et al., 2011; Pastewka et al., 2013). The choice of rock-forming asperities as a characteristic length scale is arbitrary, because grain surfaces themselves are rough down to a molecular scale, and contact between two such grains can only be partial as well; a characteristic which is at the core of fractal-like surfaces. The fact that a lattice size $\ell$ on the order of the average grain diameter is sufficient for normal contact and fluid flow modeling reflects the fact that these processes depend dominantly on large-scale roughness (Zimmerman and Bodvarsson, 1996; Vallet et al., 2009; Neuville et al., 2011). Moreover, asperities located at the surface do not deform individually upon contact, but as a continuum (Persson, 2007), due to the cohesion between them that characterizes consolidated rock. The rigid composite surface, or aperture field, that controls the elastic, frictionless contact is a function of physical processes. First, the relative shear displacement between the two initially strongly correlated surfaces controls the interfacial deformation (Borri-Brunetto et al., 1999). Secondly, surface alterations due to shear related abrasion and chemically-mediated processes may have severe effects.

If the opposing surfaces are uncorrelated, the resulting composite surface 


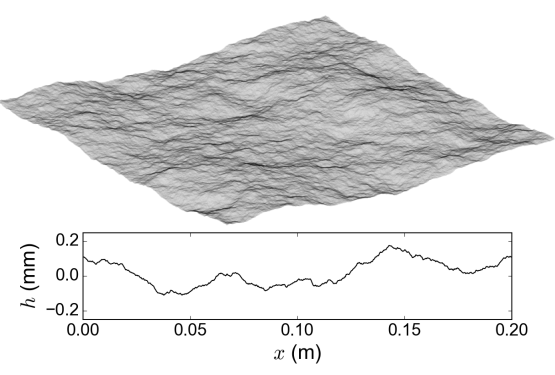

C
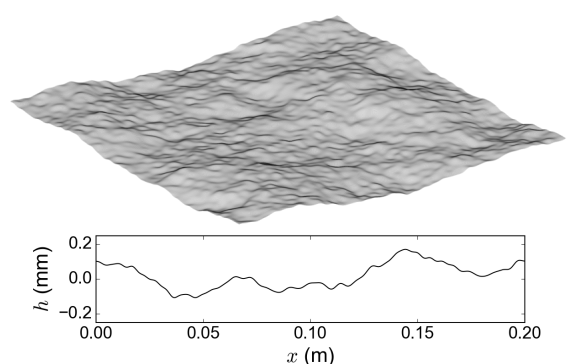

e
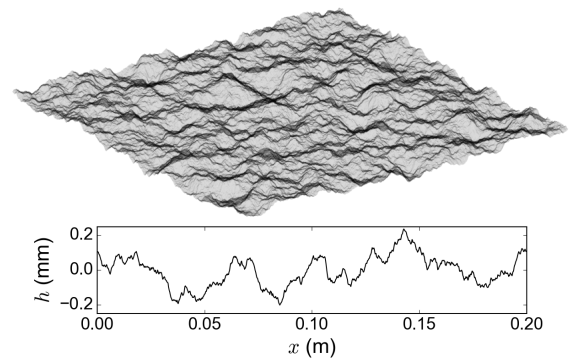

g
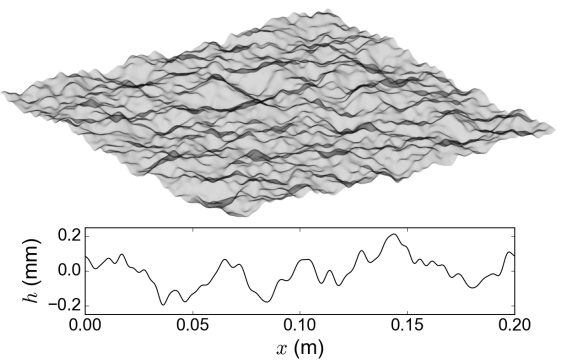
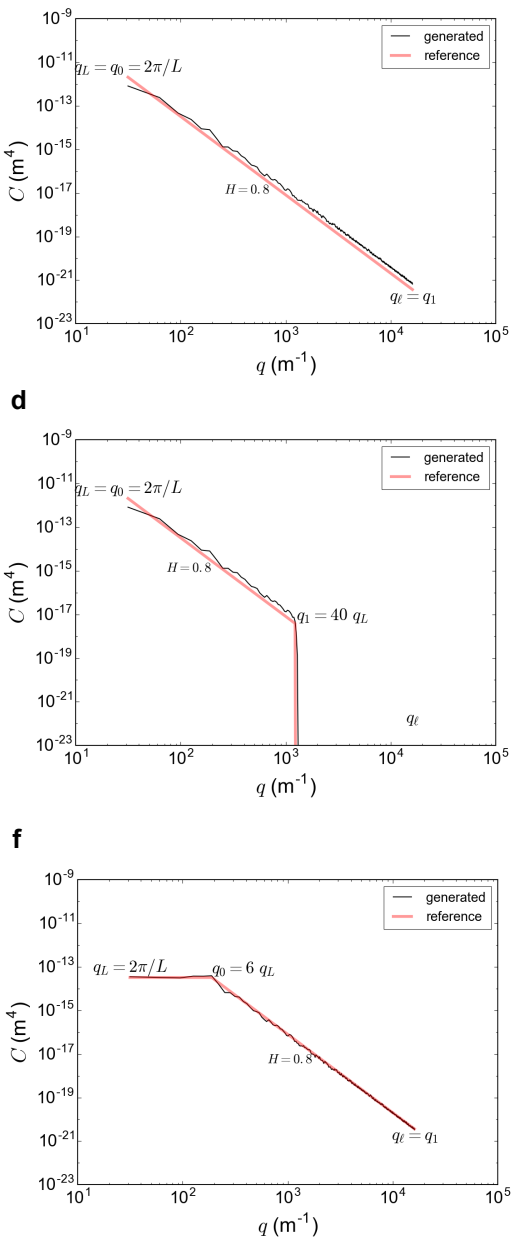

h

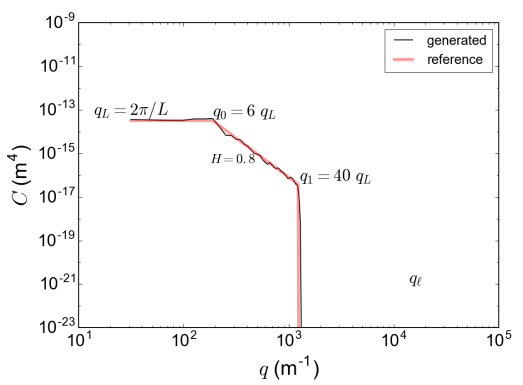

Figure 2: Illustration of cut-off $q_{1}$, roll-off $q_{0}$ and Hurst exponent $H$ for isotropic rough surfaces, their traces and the corresponding PSDs. (a-b) A surface with fractal roughness across all wavenumbers. (c-d) A surface with a roughness cut-off. (e-f) A surface with a roll-off. (g-h) A surface with both roll-off and cut-off. 


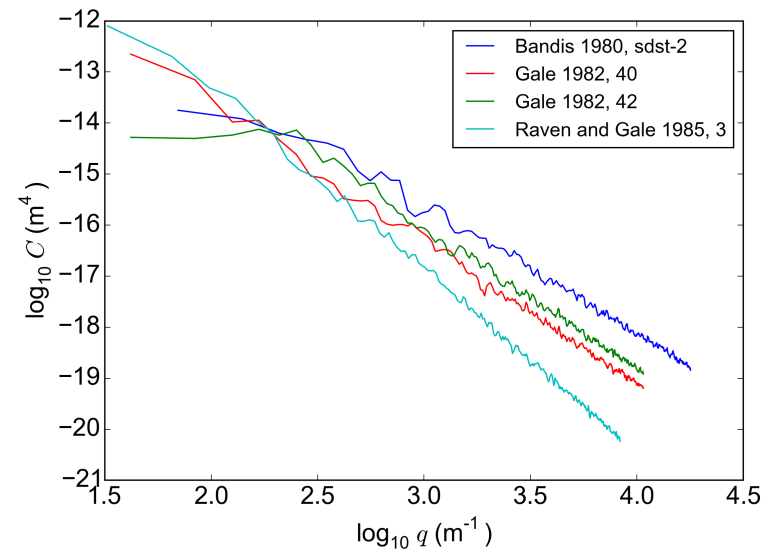

Figure 3: Radially averaged surface roughness spectra for the numerical composite surfaces generated to fit initial closure curves of Gale (1982), Bandis (1980) and Raven and Gale (1985).

has the same scaling exponent $H$ as the individual surfaces, with a reduced roughness amplitude $h_{\text {rms }}$ (Hyun et al., 2004). For opposing surfaces with shear displacement smaller than the largest roughness wavelength, the composite surface will in general be anisotropic, exhibit a roughness wavelength roll-off below the sample length, and show reduced Hurst exponents, which reflects a reduced correlation length (Brown et al., 1986; Matsuki et al., 2006).

A square composite surface can be discretized into $\mathcal{L} \times \mathcal{L}$ regular grid cells to obtain a numerical solution for contact with a planar elastic body. The method employed here is based on the solution of the displacement-pressure integrals in Fourier space embedded in a conjugate gradient algorithm (Stanley and Kato, 1997). Therein, the contact pressure is transformed to the frequency domain by a Fast Fourier Transform (FFT) algorithm, multiplied by stiffness coefficients from the Westergaard (1939) solution for contact pressure due to a sinusoidal height profile, and the resulting displacement solution is brought back to the space domain by an inverse FFT. The inherent non-linearity of the elastic contact problem that arises from the mutual dependence of contact area, contact pressure and deformation is addressed by a conjugate gradient solver. This form of algorithm (e.g. Sainsot and Lubrecht, 2011) has been used in 
numerous studies to solve the frictionless contact between rough surfaces (e.g. Almqvist et al., 2007; Sainsot and Lubrecht, 2011; Jackson and Green, 2011; Yastrebov et al., 2015).

In response to an external effective confining pressure, $p$, the obtained solution yields the contact stress, $\sigma(\boldsymbol{x})$ (Figure 4a), and the normal displacement (Figure 4e), over the fracture plane, $\boldsymbol{x}=(x, y)$ (Figure 1). A mechanical aperture field, $a_{\mathrm{m}}(\boldsymbol{x})$, is defined as the cell-wise separation between the rigid and the elastic surface (Figure 4e), with zero-apertures at points of contact. The tangent normal stiffness can be evaluated for increasing compression as

$$
\kappa(p)=\frac{\mathrm{d} p}{\mathrm{~d}\left\langle a_{\mathrm{m}}\right\rangle}
$$

where the brackets denote a spatial average. The incremental reduction in the average separation, $\mathrm{d}\left\langle a_{\mathrm{m}}\right\rangle$, is a direct measure of the additional deformation due to the presence of the fracture (Cook, 1992). The difference between the average aperture of the unstressed fracture, $\left\langle a_{\mathrm{m}}\right\rangle_{p=0}$, and the average aperture under compression, $\left\langle a_{\mathrm{m}}\right\rangle_{p}$, increases with $p$, and is commonly represented by means of closure curves (Figure 5). Numerically, Equation 3 is evaluated for discrete increments of $p$, using a second-order finite difference approximation.

The pressure dissolution model is based on a closed-form approximation to a reaction-diffusion equation in a water-quartz environment for spherical contacts (Lehner and Leroy, 2004; Bernabé and Evans, 2007), extended to arbitrarily shaped contacts (Lang et al., 2015):

$$
\frac{\mathrm{d} h}{\mathrm{~d} t}(\boldsymbol{x})=\frac{2 \frac{\Omega_{\mathrm{s}}}{k_{\mathrm{B}} T} \sigma(\boldsymbol{x})}{\frac{2}{k_{\mathrm{d}}}+\frac{\rho_{\mathrm{s}}}{\rho_{\mathrm{f}}} \frac{r_{\mathrm{d}}(\boldsymbol{x})^{2}}{4 c_{\mathrm{eq}} w D^{*}}}
$$

In Equation $4, T=150 \mathrm{~K}$ is the ambient temperature, and $r_{\mathrm{d}}(\boldsymbol{x})(\mathrm{m})$ is the diffusion distance for each point at the interface, i.e., the distance to the nearest free pore space part of the fracture. Furthermore, $\rho_{\mathrm{s}}=2650 \mathrm{~kg} \mathrm{~m}^{-3}$ is the solid density, $\rho_{\mathrm{f}}=1000 \mathrm{~kg} \mathrm{~m}^{-3}$ is the fluid density, $k_{\mathrm{d}}=2.27 \mathrm{E}-(3.826+0.002028 T$ $+4158 / T) \mathrm{m} \mathrm{s}^{-1}$ is a temperature-dependent dissolution rate constant, $\Omega_{\mathrm{s}}$ $\left(\mathrm{m}^{3}\right)$ is the molecular volume, $k_{\mathrm{B}}\left(\mathrm{J} \mathrm{K}^{-1}\right)$ is the Boltzmann constant, and $c_{\mathrm{eq}}$ $=0.055 \mathrm{E}-(0.254+1107.12 / T)$ is the equilibrium concentration in the free pore 
a

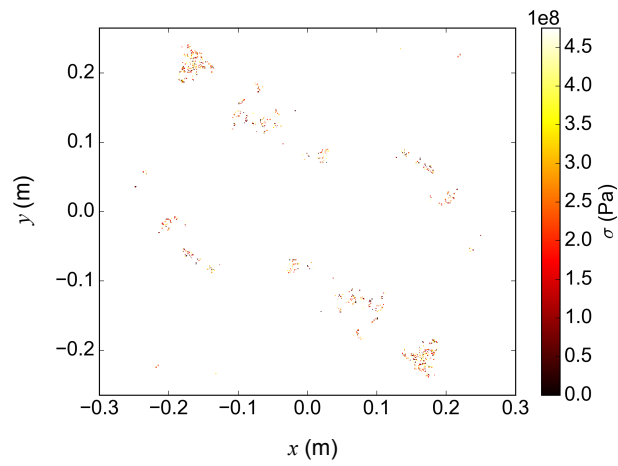

C
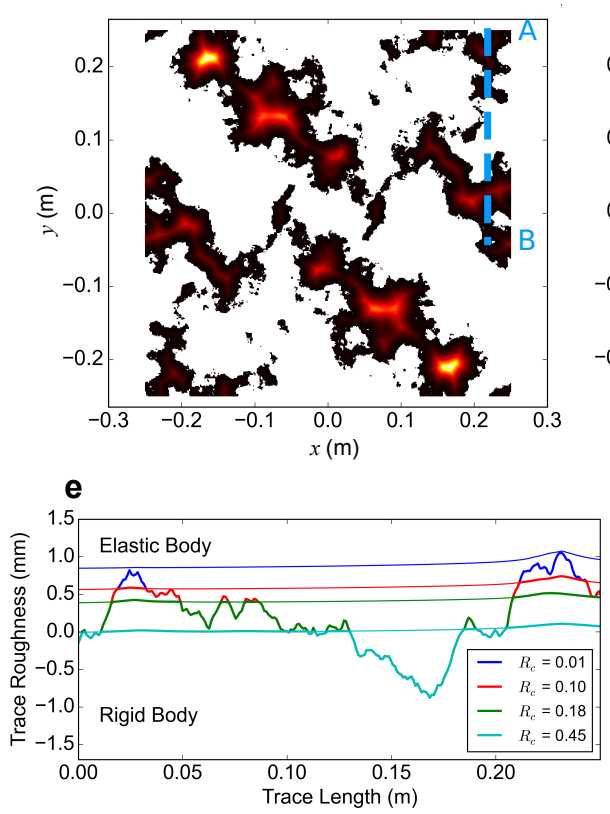

b

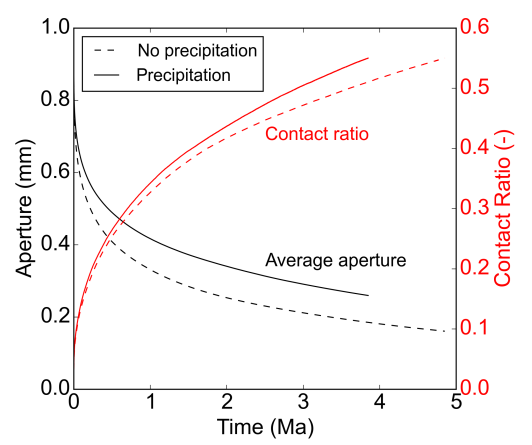

d

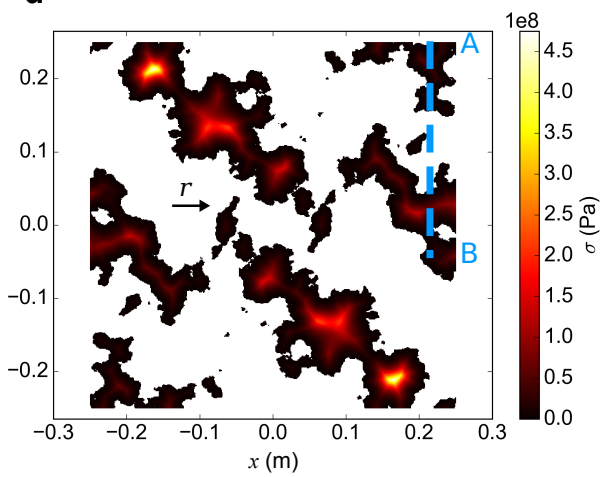

f

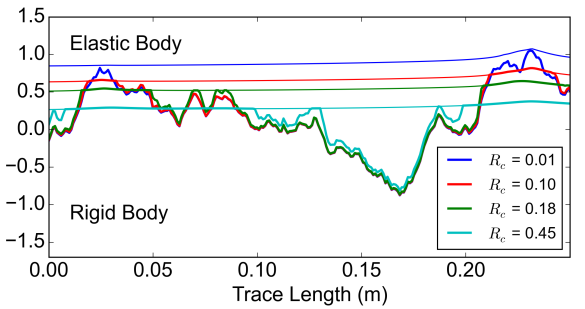

Figure 4: Qualitative and quantitative evolution of the generic fracture model at $10 \mathrm{MPa}$ and $150{ }^{\circ} \mathrm{C}$. (a) The initial profile of discrete contacts over the fracture plane, with an in situ contact ratio of $R_{\mathrm{c}}=0.01$. (b) The transient change in contact ratio and average aperture over time for the 'open' system, where only pressure solution takes place, and the 'closed' system, where dissolved mass is re-precipitated. (c) The discrete contact after 220 ka for the 'open' system, in situ $R_{\mathrm{c}}=0.21$. (d) The discrete contact after 220 ka for the 'closed' system, in situ $R_{\mathrm{c}}=0.22$. The arrow indicates an estimated void segment radius $r$. (e) A cut-plane a-b through the elastic-rigid surfaces of the 'open' system for increasing steps in time, as the in situ contact increases. (f) A cut-plane a-b through the elastic-rigid surfaces of the 'closed' system for increasing steps in time, as the in situ contact area increases. 
a

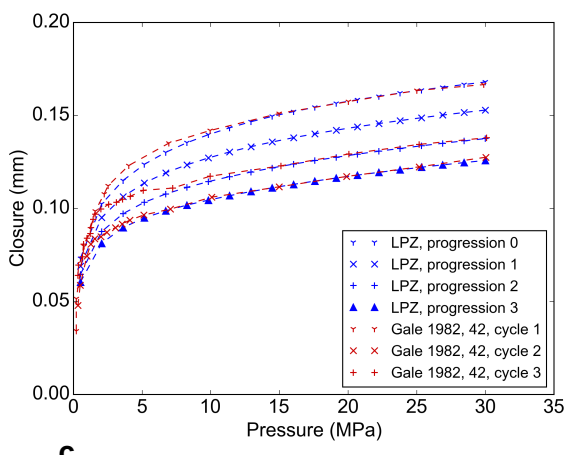

C

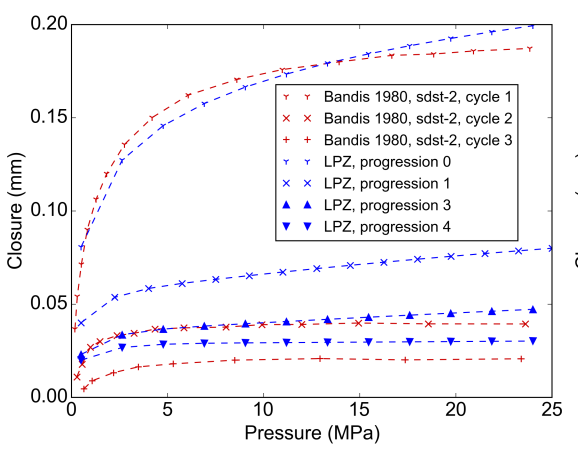

b

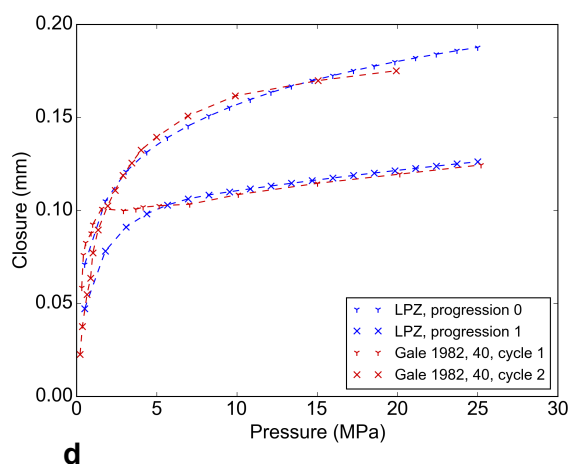

d

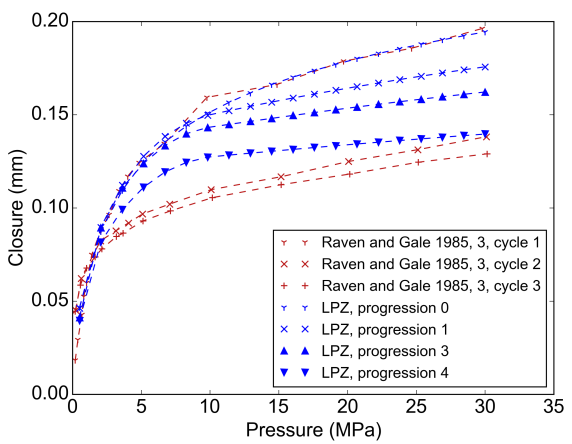

Figure 5: A comparison between multiple cycles of compression from fracture closure experiments, and the numerically obtained instantaneous fracture closure for progressing stages of pressure solution in 'open' systems. The first closure curve has been fitted to obtain surface parameters and elastic moduli for the initial numerical models. (a) Gale (1982) sample 42, (b) Gale (1982) sample 40, (c) Bandis (1980) sample sdst-2, (d) Raven and Gale (1985) sample 3. 


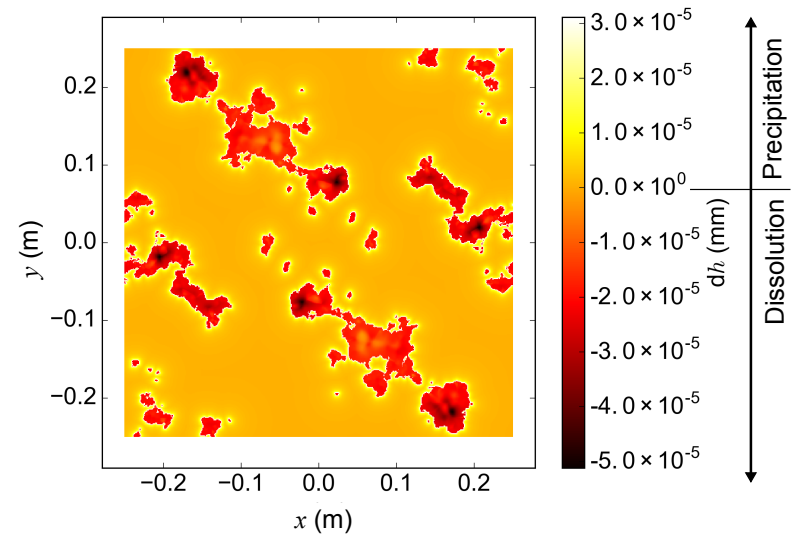

Figure 6: Surface height change $\mathrm{d} h$ for the generic fracture model under 'closed' conditions over a period of 100 years due to pressure solution and free-face precipitation.

space, expressed as mass fraction. The width of the contact-boundary diffusion zone is $w=1.0 \mathrm{E}-8 \mathrm{~m}$, and the reduced diffusion constant in this zone is $D^{*}=$ $0.1 \times 9.0 \mathrm{E}-7 \exp (15000 / R T) \mathrm{m}^{2} \mathrm{~s}^{-1}$, where $R$ is the gas constant. Note that the dissolution rate constant $k_{\mathrm{d}}$ in its convergence rate form $\left(\mathrm{m} \mathrm{s}^{-1}\right)$ is obtained by multiplying dissolution rate constants $\left(\mathrm{mol} \mathrm{m}^{-2} \mathrm{~s}^{-1}\right)$ as reported in Rimstidt and Barnes (1980) by the molar volume $\left(\mathrm{m}^{3} \mathrm{~mol}^{-1}\right)$ of Quartz.

To model precipitation of dissolved mass, the assumed scenario is that pressure solution constitutes the rate-limiting process (Weyl, 1959; Rutter, 1983), and that the pore fluid remains at its equilibrium concentration. The redistribution of dissolved silica follows a volume-conservative approximation to the diffusion-reaction problem, proportional to the inverse of the distance from the surface contacts (Figure 6). Two end-members with respect to dissolutionprecipitation have been studied. (1) 'Open' systems model pressure-solution only, and any dissolved mass is assumed to be subject to advective transport that prevents re-precipitation. (2) 'Closed' systems assume that any dissolved mass is locally conserved and precipitates near the fracture contacts in a solid volume conservative manner.

In situ conditions in terms of temperature and effective confining pressure are 
chosen as $T=423 \mathrm{~K}$ and $p=10 \mathrm{MPa}$, respectively, and are time invariable. The absolute pressure and temperature conditions are, to first order, only significant for the rate of fracture compaction, not the qualitative evolution of the pore space (Lang et al., 2015). Simulations are run until a contact ratio of $R_{\mathrm{c}} \geq 0.55$ is reached. This represents the approximate upper limit where contacting zones percolate to hydraulically seal the fracture, thus violating the 'open' system assumption.

\subsection{Surface Properties and Elastic Moduli}

Isotropic, periodic self-affine composite surfaces have been generated (Peitgen et al., 1988) for this study, discretized using $512 \times 512$ grid cells. Two different surface parameter sets have been used. The first approach studies the behavior of a generic fracture of $0.5 \times 0.5 \mathrm{~m}$ (Figure 1), where $H=0.8, \lambda_{0}=$ $L / 2, h_{\mathrm{rms}}=500 \mu \mathrm{m}$, with $60 \mathrm{GPa}$ and 0.15 for Young's modulus and Poisson's ratio, respectively. Second, based on least-squares fitting, four models have been created with surface properties and elastic moduli chosen to reproduce initial closure curves of fracture deformation experiments. These experiments have been chosen based on the classification of the fractures as being non-mated, the availability of closure data for multiple, successive loading cycles, and based on the fact that visible plastic damage has been reported over the rock surfaces as a result of the mechanical loading (Bandis, 1980; Gale, 1982; Raven and Gale, 1985). The edge length, $L$, of the created models equals the maximum dimension of the samples. The Poisson's ratio for all cases has been assumed to be 0.15 , as this parameter has a minor effect on closure curves. The fitting parameters allowed for variations in the Young's modulus, $E$, the roughness amplitude, $h_{\mathrm{rms}}$, the roughness wavelength-amplitude scaling exponent, $H$, and the roll-off wavelength, $\lambda_{0}$.

Numerical values that resulted from the fit are listed in Table 1, and radially averaged roughness power spectra (Persson et al., 2005) are shown in Figure 3. A comparison between numerical and experimental initial closure curves (Figure 5) shows good agreement for sample 42 (Gale, 1982) and sample 3 (Raven 
Table 1: Result of least-squares fit of numerical models to experimentally observed initial closure curves. The fitting variables increments are $5 \mu \mathrm{m}$ for $h_{\mathrm{rms}}, 0.1$ for $H$, power of two fractions of $L$ for $\lambda_{0}$, and $5 \mathrm{GPa}$ for $E$.

\begin{tabular}{llccccc}
\hline Author & Sample & $L \times L[\mathrm{~m}]$ & $h_{r m s}[\mu \mathrm{m}]$ & $H[-]$ & $L / \lambda_{0}[-]$ & $E[\mathrm{GPa}]$ \\
\hline Bandis (1980) & sdst-2 & $0.09 \times 0.09$ & 85 & 0.3 & 4 & 20 \\
Gale (1982) & 40 & $0.15 \times 0.15$ & 70 & 0.4 & 1 & 60 \\
& 42 & $0.15 \times 0.15$ & 60 & 0.4 & 4 & 60 \\
Raven and Gale (1985) & 3 & $0.193 \times 0.193$ & 90 & 0.8 & 1 & 65 \\
\hline
\end{tabular}

and Gale, 1985). Poorer fits have been obtained for sample 40 (Gale, 1982) and sample sdst-2 (Bandis, 1980), probably as a result of stronger correlation between the surfaces in contact, as indicated by the fast asymptotic approach towards a residual aperture with increasing load. Composite surfaces that reproduce this behavior require a more log-normal distributed surface height and anisotropic scaling exponents (e.g. Matsuki et al., 2006), but are here approximated as Gaussian and isotropic. Discrepancies can further be explained by the contributing closure mechanisms during the first cycle of compression. While the numerical model accounts for frictionless elastic contact only, the initial closure behavior in experiments is likely to also reflect gradual plastic deformation and friction-related rearrangement between the surfaces.

\section{Evolution of the Fracture Space}

After the fracturing process and subsequent shear displacement, opposing surfaces are mismatched and touch at a minuscule fraction of their nominal area, resulting in large stress concentrations at the contacts (Figure 4a). In an attempt to reach a more equilibrated state, pressure solution acts to smooth the elevated, contacting fractions of the surface. As a consequence, the initial Gaussian distribution of surface height is truncated, and small-scale roughness features in contact zones are dissolved to yield more rounded structures (Figure 1). Most notably, this effort results in (1) a more uniform distribution of 
contact pressure, (2) less pronounced stress concentrations, (3) an increase in contact area, and (4) newly formed contact regions as a result of the convergence, or approach, of the two surfaces (Figures 4c to 4f). In 'closed' systems, precipitation facilitates the growth of existing contact regions without significant convergence of the surfaces. Large aperture channels are thus preserved, while they tend to close for 'open' systems where no precipitation takes place (Figures 4e and 4f). It follows that the loss of pore-volume occurs faster in 'open' systems than in 'closed' systems (Figure 4b). At the same time, the contacting fraction of the surface is made up of a larger number of contact spots for the 'open' no-precipitation system, and of larger individual, but fewer in number contact spots for 'closed' systems with precipitation (Figures 4c and 4d). The driving force of pressure solution-driven compaction is the contact stress, which, to first order, is a function of the contact ratio, and to second order is a function of the size and roughness or curvature over the discrete contacts. Thus, the increase in contact ratio evolves in a similar manner for both systems, with precipitation acting to accelerate its increase (Figures $4 \mathrm{~b}$ ).

These changes in rock surface morphology have a profound effect on fracture stiffness, analogous to the increase in host rock bulk stiffness due to diagenetically driven cementation of the pore space (Laubach et al., 2009). Within the larger setting of a fractured rock mass and far-field stress conditions, shear fractures that experience pressure solution will be found at a high angle to filled veins, indicative of their formation at high angles with respect to the orientation of the maximum principal stress (Peacock and Sanderson, 1995). Precipitation in such shear fractures will often occur in void spaces that result from large scale pull-apart structures (Gamond, 1983). Besides their oblique orientation to the direction of shear, pull-apart connecting surfaces that experienced pressure solution are dark and braided in nature (Peacock and Sanderson, 1995). 


\section{Changes in Normal Stiffness}

The stiffness of the generic fracture in its initial state is a linear function of the applied load (Figure 7a), in agreement with empirical, numerical and theoretical models of elastic contact between randomly rough surfaces (Bandis et al., 1983; Swan, 1983; Hopkins et al., 1990; Berthoud and Baumberger, 1998; Akarapu et al., 2011). This reflects the fact that, with increasing $p$, existing contact areas grow and new contact areas form in such a way that the distributions of stress and contact spot size remain constant if normalized to the area of discrete contact (Sun et al., 1985; Persson, 2006). It follows that the contact ratio $R_{c}$ varies linearly with $p$ (Figure 8), and that the distributions of contact stress and contact patch size scale linearly with $p$ when normalized to the nominal area (Campañá et al., 2011).

The diagenetic processes studied here act to partition the nature of surface roughness over the fracture. While elevated regions are flattened, lower parts that are not in contact at in situ conditions retain their initial roughness (Figure $7 \mathrm{~b})$. The instantaneous closure of such a fracture in its chemically altered state gives rise to two contact regimes as a function of normal load. At loads roughly equal to or below in situ conditions, stiffening of the fracture is controlled by smooth, low curvature features that result from dissolution-precipitation at and near contacts. Over these parts of the surface, a minimal increase in compression yields a disproportionately large increase in contact area. At higher loads, incremental contact occurs over the unaltered, self-affine rough fraction of the surfaces. Any incremental increase in contact area thus requires a much larger load increment over this part of the surface. The closure of the fracture, and the resulting stiffness curve, reflects these two regimes (Figure 7a). Stiffening transitions from approximately exponential to approximately linear at a load slightly larger than the $10 \mathrm{MPa}$ effective confining pressure under which compaction is modeled. It follows that this in situ pressure roughly marks the transition in contact from diagenetically rounded to initially rough surface fractions. The notion that the free fracture surfaces tend to retain their self-affine 
roughness nature during chemical alterations has also been shown to extend to poly- and mono-mineralic fractures for dissolution dominated processes (Gouze et al., 2003; Noiriel et al., 2013).

The normal stiffness magnitude increases over the entire load spectrum as a result of these mechanical-chemical processes. Primarily, this reflects the reduced amount of normal load required to generate additional contact area in the low load stage, and the observed increase in contact ratio at all loads. Furthermore, reduced stress concentrations over smoothened contacts result in reduced elastic compression of the contacts. Shortened free wall intervals that result from growing and newly formed contact spots reduce the deflection of surfaces into the fracture void space. Combined with the increased strain energy accommodated in larger contact structures, the elastic compression of the pore-space is much reduced compared to the fresh fracture, thus increasing its stiffness. At large loads, contact is made over fractions of the surface least affected from diagenetic processes, thus the difference in magnitude between initial and altered fracture stiffness is lessened.

For both the 'open' system that models pressure solution only, and the 'closed' system that models re-precipitation of the dissolved mass, the obtained instantaneous stiffness curves for points in time of equal in situ-contact ratios have been found to evolve in a similar manner (Figure 8b). A higher stiffness throughout the load spectrum is obtained for the 'open' system. This reflects the increased convergence of the opposing surfaces, and the fact that the contacting fraction of the surface is made up of a larger number of individual contact patches, as opposed to fewer in number but individually larger patches for the 'closed' system with precipitation. Also, 'closed' systems tend to retain deep channels, which, along with the larger separation between contact points, allow for larger elastic deformation into the void space. These observations are in line with experimental (Kendall and Tabor, 1971) and numerical results (Hopkins et al., 1987, 1990) that relate reduced distance between contacts to higher interfacial stiffness. It can be concluded that the stiffness of the presented models is mainly a function of the magnitude of the in situ contact ratio (e.g. 

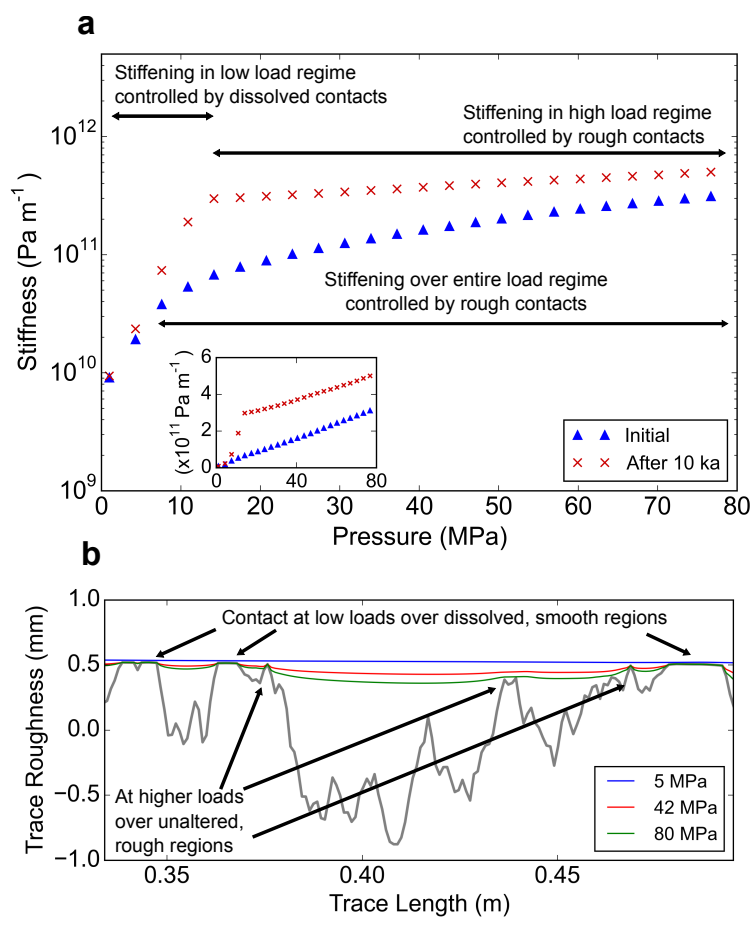

Figure 7: Quantitative and qualitative illustration of the observed change in normal stiffness for the fracture model. (a) Instantaneous normal stiffness curves for the initial fracture, and after $10 \mathrm{ka}$ of pressure solution and free-face precipitation. Inset: Linear plot. (b) A cutplane through the surface of the fracture model after $10 \mathrm{ka}$ for three steps of increasing load, illustrating instantaneous closure behavior of the altered fracture. 
Petrovitch et al., 2013, 2014), and to a slightly lesser degree a response to the size and number of contact patches that result from whether or not dissolved mass precipitates locally. From this perspective, the non-linearity of stiffness follows the change in contact area-load relationship, from classic linear to an exponential-linear function (Figure 8a).

\section{Similarities to Closure Behavior due to Plastic Damage}

With increasing normal loads, irreversible mechanisms contribute to the closure of a rock fracture, in particular if the surfaces in contact are non-mated (Bandis, 1980). For one, contacting fractions subjected to large stress concentrations will experience plastic yield. Furthermore, minor lateral shifts will occur as local shear forces at inclined contacts exceed the frictional resistance. The observed flattening of tips caused by plastic yield (Brown and Scholz, 1986), however, should be expected to have similar effects on fracture closure as does the process of pressure solution.

Qualitatively, the hysteresis observed during multiple closure cycles has two main characteristics: (1) A reduction in residual aperture, and (2) this residual is reached at lower loads than during the initial loading (Bandis, 1980; Gale, 1982; Raven and Gale, 1985). Numerically-obtained closure curves of fractures with increasing degree of pressure solution damage are consistent with this behavior (Figure 5). Although there is a pronounced mismatch for sample 3 (Raven and Gale, 1985), good agreement is observed between numerical and experimental closure curves for the other comparisons. This suggests that closure of fractures subjected to pressure solution and plastic damage is governed by the same firstorder mechanism, namely the partitioning of the fracture surface into a flattened, elevated fraction, and a lower one that retains its initial roughness.

\section{Fracture Closure and Stiffness-Compliance Relationships}

Noise in experimentally measured closure data makes direct differentiation with respect to pressure difficult, see Equation 3. Stiffness, therefore, is of- 


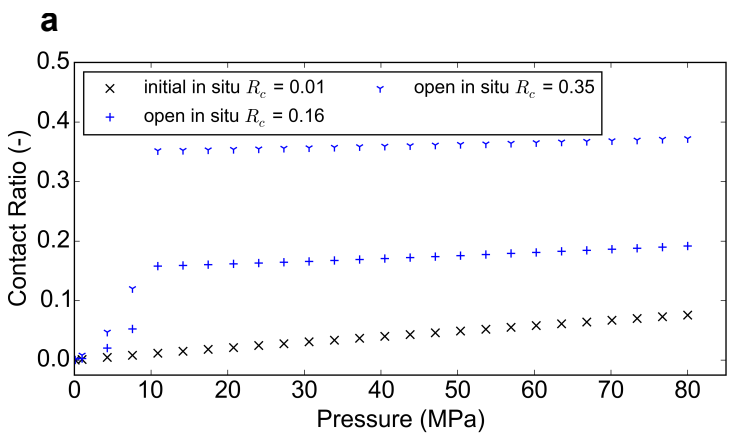

b

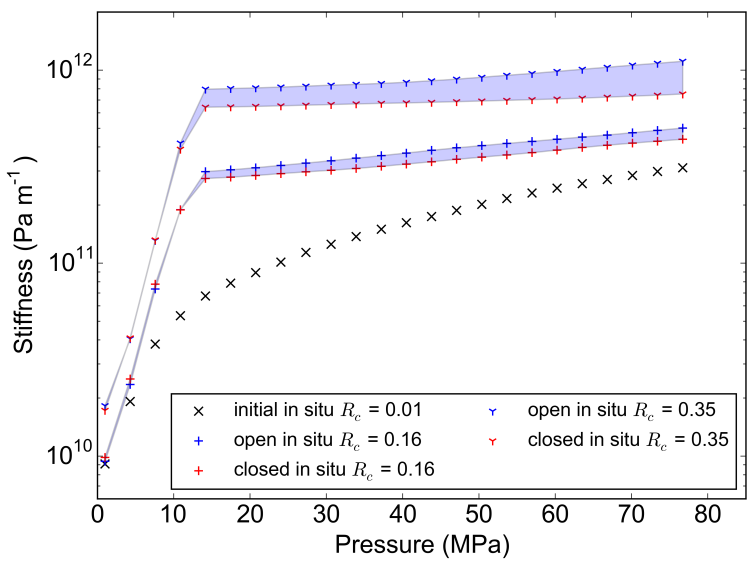

Figure 8: Instantaneous contact ratio and stiffness as a function of pressure for the generic fracture model at its initial state and after progressing stages of pressure solution and precipitation have altered the fracture. (a) The instantaneous contact ratio as a function of pressure for the initial fracture and after pressure solution has acted for 1.2 Ma to increase the in-situ fractional contact area $R_{\mathrm{c}}$ under $10 \mathrm{MPa}$ to 16 and 35 percent. (b) The instantaneous normal stiffness of the initial fracture and after pressure solution only ('open') and pressure solution and precipitation ('closed') have led to an increase of the in-situ fractional contact area $R_{\mathrm{C}}$ to 16 and 35 percent under $10 \mathrm{MPa}$. 
ten evaluated on smooth curve-fits (e.g. Zangerl et al., 2008). Least-squares fits of the numerically-obtained closure data (Figure 9a) of the generic $0.5 \times 0.5$ $\mathrm{m}$ fracture have been generated for two functional models of fracture normal deformation; a hyperbolic and a logarithmic relationship. The closure models proposed by Bandis (1980) and Goodman (1976) can be generalized to a hyperbolic relationship of the form (Muralha, 1999)

$$
\delta_{\mathrm{n}}=\frac{\sigma_{\mathrm{n}}^{m}}{a+b \sigma_{\mathrm{n}}^{m}}
$$

where $\delta_{\mathrm{n}}(\mathrm{m})$ is the fracture closure, i.e., $\delta_{\mathrm{n}}=\left\langle a_{\mathrm{m}}\right\rangle_{p=0}-\left\langle a_{\mathrm{m}}\right\rangle_{p}$ in this study, $\sigma_{\mathrm{n}}(\mathrm{Pa})$ is the nominal normal stress acting on the fracture, i.e., the confining pressure $p$, and $a, b$ and $m$ are fitting variables. The Bandis model is a special case of Equation 5 when $m=1$, and the Goodman model can be expressed by Equation 5 beyond a shift due to the initial seating stress (Muralha, 1999). Besides a hyperbolic relationship, closure models for mismatched joints have also been expressed as logarithmic, or exponential relationships (Goodman, 1976; Detournay, 1979; Bandis et al., 1983). A two-parameter form was proposed by Detournay (1979)

$$
\delta_{\mathrm{n}}=a \ln \left(\frac{\sigma_{\mathrm{n}}}{b}+1\right)
$$

Both the hyperbolic and logarithmic model provide good fits to the closure curve of the initial fracture model (Figure 9a), with correlation coefficients of $R$ $=0.996$ and $R=0.998$, respectively. The fit of both models to the instantaneous closure of the fracture in its state after $10 \mathrm{ka}$ of pressure solution is nearly identical, with $R=0.989$. This poorer fit is most pronounced over the low-load regime between 5 and $10 \mathrm{MPa}$, where closure transitions from being governed by the dissolved surface regions to being governed by the rough surface regions. Similar discrepancies can be expected when fitting smooth curves to the closure data of cycle 2 and 3, sample 42 (Figure 5a), and cycle 2, sample 40 (Figure $5 b)$, of Gale (1982).

The misfit of smooth curves to the closure of the dissolved fracture in the low load regime has a profound effect on the nature of the obtained stiffness curves (Figure 9b). A smooth increase in stiffness entirely masks the transition from 
rapid stiffening over dissolved contacts, to a much reduced stiffening over the rough surface fraction; for a comparison see Figure 9a for the stiffness derived from the curve fit, and Figure 7a for stiffness derived directly from numerical closure data. For the logarithmic closure model, Equation 6, normal stiffness is a linear function of pressure. The hyperbolic model, Equation 5, results in sublinear stiffness for the initial fracture, and superlinear stiffness of the fracture after dissolution, thus bounding the stiffness values obtained from the logarithmic fit at larger loads. Stiffness values obtained from direct differentiation of the numerical closure data (Figure $7 \mathrm{a}$ ) are closer to the logarithmic fit in each case.

Analytic models of closure often rely on the idealization of the fracture as a simplified shape, and the stiffness results from elastic compression of the void space. With the exception of Zimmerman (2008), these models do not account for incremental increase in contact ratio with pressure, which constitutes an intrinsic link to fracture normal stiffness (e.g. Petrovitch et al., 2014). For isolated open penny-shaped cracks of radius $r$, normal stiffness can be expressed as (Sayers and Kachanov, 1995)

$$
\kappa_{\mathrm{n}}=\frac{1}{B_{\mathrm{N}}}=\frac{3 \pi E}{16 r\left(1-\nu^{2}\right)}
$$

where $B_{\mathrm{N}}\left(\mathrm{m} \mathrm{Pa}^{-1}\right)$ is the normal compliance of the crack. When the fracture is conceptualized as a collection of collinear elliptical cracks (Myer, 2000), its normal stiffness can be approximated for large contact ratios as (Jaeger et al., 2007, p. 371)

$$
\kappa_{n} \approx \frac{2 G}{\pi r(1-\nu)(1-c)}=\frac{E}{\pi r\left(1-\nu^{2}\right)(1-c)}
$$

where $r(\mathrm{~m})$ is the half-length of the elliptical cracks, $\nu(-)$ and $E(\mathrm{~Pa})$ are, respectively, the Poisson's ration and Young's modulus of the intact rock, and $c$ (-) is the contact ratio, i.e., $c=R_{\mathrm{c}}$. The accuracy of the approximation in Equation 7 should be expected to increase with progressing pressure solution and precipitation, due to the increase in fractional contact area. This is accompanied by an increased separation between void space segments, which reduces their mutual influence on deformation and thus renders them more and more 
mechanically independent. For the generic fracture model under 'closed' conditions after 1.2 Ma, an in situ contact ratio of 0.35 (Figure 4b), an estimated radius of void space segments of $r=0.05 \mathrm{~m}$ (Figure 4d), with $E=60 \mathrm{GPa}$, Equation 8 yields $\kappa_{\mathrm{n}}=6.0110^{11} \mathrm{~Pa} \mathrm{~m}^{-1}$, comparable to the range of 6-7 $10^{11}$ $\mathrm{Pa} \mathrm{m}^{-1}$ obtained numerically for $p>10 \mathrm{MPa}$ (Figure $8 \mathrm{~b}$ ). The good agreement affirms that larger patches of void space deform increasingly independent of each other as the fracture compacts due to pressure solution. The use of Equation 7 alone leads to a slight underestimation of the compliance of the fracture if $r=$ $0.05 \mathrm{~m}$ of a single void segment is used, in which case $\kappa_{\mathrm{n}}=7.210^{11} \mathrm{~Pa} \mathrm{~m}^{-1}$.

\section{Conclusions}

It has long been established that characteristics of the structural process that result in the formation of fractures are directly linked to their stiffness. Primarily, the size of the initial fracture, and its subsequent shear displacement, govern the magnitude and evolution of fracture closure as a function of pressure. The presented findings make a strong case that the history of in situ-conditions has similarly profound implications, through diagenetic processes that change the morphology of the surfaces in contact. For the modeled processes of pressure solution and precipitation, the key mechanism causing the general increase in stiffness is the growth and leveling of contact zones due to pressure solution, which decreases free wall deflection and the extent to which contacting asperities are compressed. Apart from an increase in magnitude, a distinct change in the nature of the normal stiffness curve is observed. The in situ-confining pressure of the fracture marks a transition from rapid, exponential-like, towards approximately linear stiffening with increasing load. This reflects the diagenetically induced surface roughness partitioning of the fracture surface. Elevated regions in contact are smooth, well rounded to flat, as a result of dissolution and precipitation. These regions control fracture closure at low loads. Regions of the surfaces that are not in contact under in situ-conditions retain their self-affine rough nature, and control the incremental increase in stiffness at high loads. 


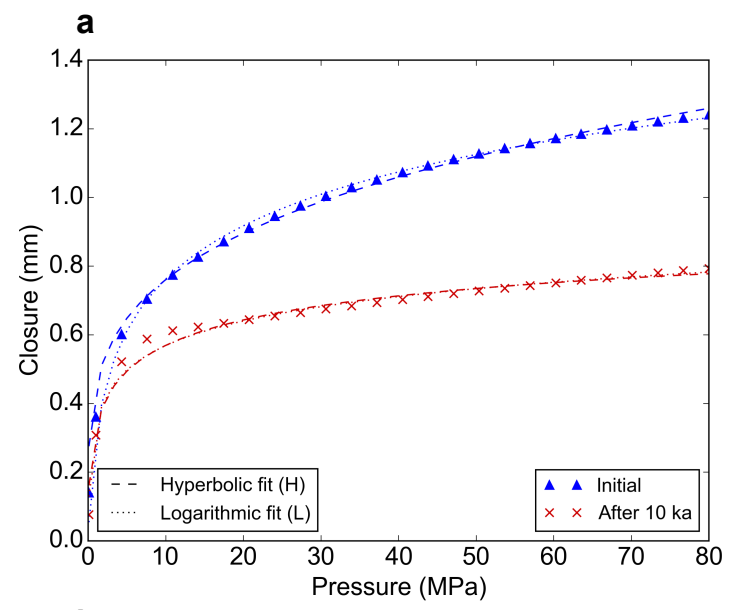

b

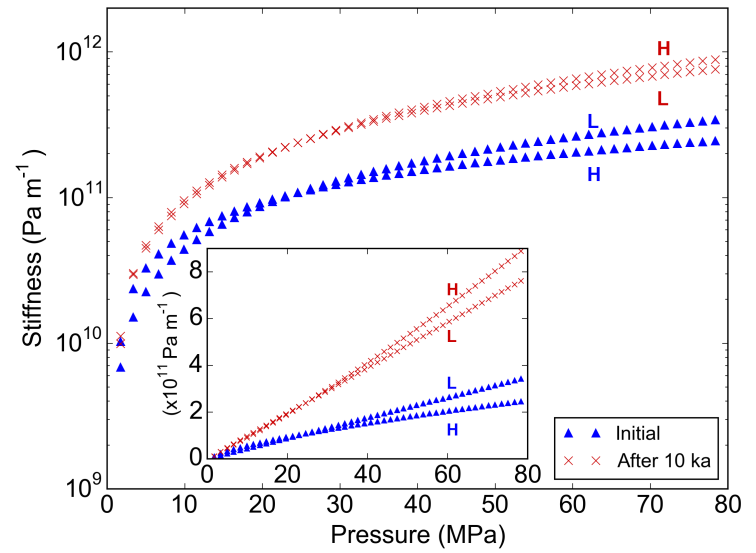

Figure 9: Stiffness derived from closure curve-fits for the generic fracture model. (a) Instantaneous closure data and curve fit of the initial fracture and the fracture after $10 \mathrm{ka}$ of pressure solution and precipitation. (b) Stiffness derived from fitted closure curves for the hyperbolic (H) and logarithmic (L) model. Inset: Linear plot. 
Similar to plastic yield at contacting asperities, a decrease in initial aperture results, and the residual aperture is reached at a much lower level of compression. While the presented model accounts only for pressure solution and free-face precipitation, other diagenetic processes, such as stress corrosion (Atkinson, 1980) or contact overgrowth (Beeler and Hickman, 2015), should be expected to affect stiffness in a similar manner.

\section{Acknowledgments}

This work was conducted within the international DECOVALEX Project (http://www.decovalex.org), with funding from Radioactive Waste Management Limited (RWM) (http://www.nda.gov.uk/rwm), a wholly-owned subsidiary of the Nuclear Decommissioning Authority. The authors also thank the European Commission for partially funding this work through the TRUST Collaborative Project, 309067.

\section{References}

\section{References}

Akarapu, S., Sharp, T., Robbins, M.O., 2011. Stiffness of contacts between rough surfaces. Physical Review Letters 106, 204301. 1011.1479.

Almqvist, A., Sahlin, F., Larsson, R., Glavatskih, S., 2007. On the dry elastoplastic contact of nominally flat surfaces. Tribology International 40, 574-579.

Atkinson, B.K., 1980. Stress corrosion and the rate-dependent tensile failure of a fine-grained quartz rock. Tectonophysics 65, 281-290.

Bandis, S.C., 1980. Experimental studies of scale effects on shear strength, and deformation of rock joints. Phd thesis. University of Leeds.

Bandis, S.C., Lumsden, A.C., Barton, N.R., 1983. Fundamentals of rock joint deformation. International Journal of Rock Mechanics and Mining Sciences \& Geomechanics Abstracts 20, 249-268. 
Barton, C.A., Zoback, M.D., Moos, D., 1995. Fluid flow along potentially active faults in crystalline rock. Geology 23, 683-686.

Beeler, N.M., Hickman, S.H., 2015. Direct measurement of asperity contact growth in quartz at hydrothermal conditions. Journal of Geophysical Research: Solid Earth 120, 3599-3616.

Bernabé, Y., Evans, B., 2007. Numerical modelling of pressure solution deformation at axisymmetric asperities under normal load. Geological Society, London, Special Publications 284, 185-205.

Berthoud, P., Baumberger, T., 1998. Shear stiffness of a solid-solid multicontact interface. Proceedings of the Royal Society A: Mathematical, Physical and Engineering Sciences 454, 1615-1634.

Borri-Brunetto, M., Carpinteri, A., Chiaia, B., 1999. Scaling phenomena due to fractal contact in concrete and rock fractures. International Journal of Fracture 12, 221-238.

Brown, S.R., Kranz, R.L., Bonner, B.P., 1986. Correlation between the surfaces of natural rock joints. Geophysical Research Letters 13, 1430-1433.

Brown, S.R., Scholz, C.H., 1985. Closure of random elastic surfaces in contact. Journal of Geophysical Research 90, 5531.

Brown, S.R., Scholz, C.H., 1986. Closure of rock joints. Journal of Geophysical Research 91, 4939.

Campañá, C., Persson, B.N.J., Müser, M.H., 2011. Transverse and normal interfacial stiffness of solids with randomly rough surfaces. Journal of Physics: Condensed Matter 23, 085001.

Candela, T., Renard, F., Klinger, Y., Mair, K., Schmittbuhl, J., Brodsky, E.E., 2012. Roughness of fault surfaces over nine decades of length scales. Journal of Geophysical Research 117, B08409. 
Chester, F.M., Logan, J.M., 1986. Implications for mechanical properties of brittle faults from observations of the Punchbowl fault zone, California. Pure and Applied Geophysics PAGEOPH 124, 79-106.

Cook, N.G.W., 1992. Natural joints in rock: Mechanical, hydraulic and seismic behaviour and properties under normal stress. International Journal of Rock Mechanics and Mining Sciences \& Geomechanics Abstracts 29, 198-223.

Detournay, E., 1979. The interaction of deformation and hydraulic conductivity in rock fracture: An experimental and analytical study. Msc thesis. University of Minnesota.

Durham, W.B., Bourcier, W.L., Burton, E.A., 2001. Direct observation of reactive flow in a single fracture. Water Resources Research 37, 1-12.

Fardin, N., Feng, Q., Stephansson, O., 2004. Application of a new in situ 3D laser scanner to study the scale effect on the rock joint surface roughness. International Journal of Rock Mechanics and Mining Sciences 41, 329-335.

Fardin, N., Stephansson, O., Jing, L., 2001. The scale dependence of rock joint surface roughness. International Journal of Rock Mechanics and Mining Sciences 38, 659-669.

Gale, J.E., 1982. The effects of fracture type (induced versus natural) on the stress-fracture closure-fracture permeability relationships. The 23rd US Symposium on Rock Mechanics, 290-298.

Gamond, J.F., 1983. Displacement features associated with fault zones: a comparison between observed examples and experimental models. Journal of Structural Geology 5, 33-45.

Gamond, J.F., 1987. Bridge structures as sense of displacement criteria in brittle fault zones. Journal of Structural Geology 9, 609-620.

Goodman, R.E., 1976. Methods of Geological Engineering in Discontinuous Rocks. West Publishing, New York, NY. 
Gouze, P., Noiriel, C., Bruderer, C., Loggia, D., Leprovost, R., 2003. X-ray tomography characterization of fracture surfaces during dissolution. Geophysical Research Letters 30.

Gratier, J.P., Gamond, J.F., 1990. Transition between seismic and aseismic deformation in the upper crust. Geological Society, London, Special Publications 54, 461-473.

Gratz, A.J., 1991. Solution-transfer compaction of quartzites: Progress toward a rate law. Geology 19, 901.

Greenwood, J.A., Williamson, J.B.P., 1966. Contact of nominally flat surfaces. Proceedings of the Royal Society A: Mathematical, Physical and Engineering Sciences 295, 300-319.

Hopkins, D.L., Cook, N.G.W., Myer, L.M., 1987. Fracture Stiffness and Aperture as a Function of Applied Stress and Contact Geometry. 28th US Symposium on Rock Mechanics, 673-680.

Hopkins, D.L., Cook, N.G.W., Myer, L.R., 1990. Normal joint stiffness as a function of spatial geometry and surface roughness, in: Barton, Stephansson (Eds.), Rock Joints. Balkema, Rotterdam, pp. 203-210.

Hyun, S., Pei, L., Molinari, J.F., Robbins, M., 2004. Finite-element analysis of contact between elastic self-affine surfaces. Physical Review E 70, 026117.

Jackson, R.L., Green, I., 2011. On the modeling of elastic contact between rough surfaces. Tribology Transactions 54, 300-314.

Jaeger, J.C., Cook, N.G.W., Zimmerman, R.W., 2007. Fundamentals of Rock Mechanics. Blackwell. 4th edition.

Kendall, K., Tabor, D., 1971. An ultrasonic study of the area of contact between stationary and sliding surfaces. Proceedings of the Royal Society A: Mathematical, Physical and Engineering Sciences 323, 321-340. 
Lander, R.H., Laubach, S.E., 2015. Insights into rates of fracture growth and sealing from a model for quartz cementation in fractured sandstones. Geological Society of America Bulletin 127, 516-538.

Lang, P.S., Paluszny, A., Zimmerman, R.W., 2015. Hydraulic sealing due to pressure solution contact zone growth in siliciclastic rock fractures. Journal of Geophysical Research: Solid Earth 120, 4080-4101.

Laubach, S.E., 2003. Practical approaches to identifying sealed and open fractures. AAPG Bulletin 87, 561-579.

Laubach, S.E., Olson, J.E., Gale, J.F., 2004. Are open fractures necessarily aligned with maximum horizontal stress? Earth and Planetary Science Letters 222, 191-195.

Laubach, S.E., Olson, J.E., Gross, M.R., 2009. Mechanical and fracture stratigraphy. AAPG Bulletin 93, 1413-1426.

Lehner, F., Leroy, Y., 2004. Sandstone compaction by intergranular pressure solution, in: Guéguen, Y., Boutéca, M. (Eds.), Mechanics of Fluid-Saturated Rocks. International Geophysics Series. volume 89, pp. 115-168.

Luan, B., Robbins, M.O., 2005. The breakdown of continuum models for mechanical contacts. Nature 435, 929-932.

Matsuki, K., Chida, Y., Sakaguchi, K., Glover, P.W.J., 2006. Size effect on aperture and permeability of a fracture as estimated in large synthetic fractures. International Journal of Rock Mechanics and Mining Sciences 43, 726-755.

Moore, D.E., Lockner, D.A., Byerlee, J.D., 1994. Reduction of permeability in granite at elevated temperatures. Science 265, 1558-61.

Morrow, C.A., Moore, D.E., Lockner, D.A., 2001. Permeability reduction in granite under hydrothermal conditions. Journal of Geophysical Research 106, 30551. 
Mourzenko, V., Galamay, O., Thovert, J.F., Adler, P., 1997. Fracture deformation and influence on permeability. Physical Review E 56, 3167-3184.

Muralha, J., 1999. A probabilistic model for the normal compliance of rock joints, in: 9th ISRM Congress, 25-28 August, Paris, France, International Society for Rock Mechanics.

Myer, L.R., 2000. Fractures as collections of cracks. International Journal of Rock Mechanics and Mining Sciences 37, 231-243.

Nayak, P.R., 1971. Random process model of rough surfaces. Journal of Lubrication Technology 93, 398.

Neuville, A., Toussaint, R., Schmittbuhl, J., 2011. Hydraulic transmissivity and heat exchange efficiency of open fractures: a model based on lowpass filtered apertures. Geophysical Journal International 186, 1064-1072.

Noiriel, C., Gouze, P., Madé, B., 2013. 3D analysis of geometry and flow changes in a limestone fracture during dissolution. Journal of Hydrology 486, 211-223.

Pastewka, L., Prodanov, N., Lorenz, B., Müser, M.H., Robbins, M.O., Persson, B.N.J., 2013. Finite-size scaling in the interfacial stiffness of rough elastic contacts. Physical Review E - Statistical, Nonlinear, and Soft Matter Physics 87, 1-9. 1210.4635.

Peacock, D.C.P., Sanderson, D.J., 1995. Pull-aparts, shear fractures and pressure solution. Tectonophysics 241, 1-13.

Peitgen, H.O., Saupe, D., Barnsley, M.F., Fisher, Y., McGuire, M., 1988. The science of fractal images. Springer New York.

Persson, B., 2007. Relation between Interfacial Separation and Load: A General Theory of Contact Mechanics. Physical Review Letters 99, 125502.

Persson, B.N.J., 2001. Theory of rubber friction and contact mechanics. Journal of Chemical Physics 115, 3840. 
Persson, B.N.J., 2006. Contact mechanics for randomly rough surfaces. Surface Science Reports 61, 201-227. 0510232.

Persson, B.N.J., 2014. On the fractal dimension of rough surfaces. Tribology Letters 54, 99-106.

Persson, B.N.J., Albohr, O., Tartaglino, U., Volokitin, A.I., Tosatti, E., 2005.

On the nature of surface roughness with application to contact mechanics, sealing, rubber friction and adhesion. Journal of Physics: Condensed Matter 17, R1-R62. 0502419v1.

Petrovitch, C.L., Nolte, D.D., Pyrak-Nolte, L.J., 2013. Scaling of fluid flow versus fracture stiffness. Geophysical Research Letters 40, 2076-2080.

Petrovitch, C.L., Pyrak-Nolte, L.J., Nolte, D.D., 2014. Combined scaling of fluid flow and seismic stiffness in single fractures. Rock Mechanics and Rock Engineering 47, 1613-1623.

Polak, A., Elsworth, D., Yasuhara, H., Grader, A.S., Halleck, P.M., 2003. Permeability reduction of a natural fracture under net dissolution by hydrothermal fluids. Geophysical Research Letters 30, 2020.

Poon, C.Y., Sayles, R.A., Jones, T.A., 1992. Surface measurement and fractal characterization of naturally fractured rocks. Journal of Physics D: Applied Physics 25, 1269-1275.

Power, W.L., Tullis, T.E., Brown, S.R., Boitnott, G.N., Scholz, C.H., 1987. Roughness of natural fault surfaces. Geophysical Research Letters 14, 29.

Pyrak-Nolte, L.J., Morris, J.P., 2000. Single fractures under normal stress: The relation between fracture specific stiffness and fluid flow. International Journal of Rock Mechanics and Mining Sciences 37, 245-262.

Pyrak-Nolte, L.J., Myer, L.R., Cook, N.G.W., 1990. Transmission of seismic waves across single natural fractures. Journal of Geophysical Research 95, 8617. 
Raven, K.G., Gale, J.E., 1985. Water flow in a natural rock fracture as a function of stress and sample size. International Journal of Rock Mechanics and Mining Sciences \& Geomechanics Abstracts 22, 251-261.

Rimstidt, J., Barnes, H., 1980. The kinetics of silica-water reactions. Geochimica et Cosmochimica Acta 44, 1683-1699.

Rutter, E.H., 1976. The kinetics of rock deformation by pressure solution [and discussion]. Philosophical Transactions of the Royal Society A: Mathematical, Physical and Engineering Sciences 283, 203-219.

Rutter, E.H., 1983. Pressure solution in nature, theory and experiment. Journal of the Geological Society 140, 725-740.

Sainsot, P., Lubrecht, A.A., 2011. Efficient solution of the dry contact of rough surfaces: a comparison of fast Fourier transform and multigrid methods. Proceedings of the Institution of Mechanical Engineers, Part J: Journal of Engineering Tribology 225, 441-448.

Sayers, C.M., Kachanov, M., 1995. Microcrack-induced elastic wave anisotropy of brittle rocks. Journal of Geophysical Research 100, 4149.

Sayers, C.M., Taleghani, A.D., Adachi, J., 2009. The effect of mineralization on the ratio of normal to tangential compliance of fractures. Geophysical Prospecting 57, 439-446.

Schmittbuhl, J., Schmitt, F., Scholz, C.H., 1995. Scaling invariance of crack surfaces. Journal of Geophysical Research 100, 5953-5973.

Stanley, H.M., Kato, T., 1997. An FFT-Based method for rough surface contact. Journal of Tribology 119, 481.

Sun, Z., Gerrard, C., Stephansson, O., 1985. Rock joint compliance tests for compression and shear loads. International Journal of Rock Mechanics and Mining Sciences \& Geomechanics Abstracts 22, 197-213. 
Swan, G., 1983. Determination of stiffness and other joint properties from roughness measurements. Rock Mechanics and Rock Engineering 16, 19-38.

Tatone, B.S.A., Grasselli, G., 2013. An investigation of discontinuity roughness scale dependency using high-Resolution surface measurements. Rock Mechanics and Rock Engineering 46, 657-681.

Vallet, C., Lasseux, D., Zahouani, H., Sainsot, P., 2009. Sampling effect on contact and transport properties between fractal surfaces. Tribology International 42, 1132-1145.

Westergaard, H.M., 1939. Bearing pressures and cracks. Journal of Applied Mechanics 61, A49-A53.

Weyl, P.K., 1959. Pressure solution and the force of crystallization: a phenomenological theory. Journal of Geophysical Research 64, 2001-2025.

Yang, C., Tartaglino, U., Persson, B.N.J., 2006. A multiscale molecular dynamics approach to contact mechanics. The European Physical Journal E: Soft Matter 19, 47-58. 0508264v3.

Yastrebov, V.A., Anciaux, G., Molinari, J.F., 2015. From infinitesimal to full contact between rough surfaces: Evolution of the contact area. International Journal of Solids and Structures 52, 83-102.

Yasuhara, H., Polak, A., Mitani, Y., Grader, A., Halleck, P., Elsworth, D., 2006. Evolution of fracture permeability through fluidrock reaction under hydrothermal conditions. Earth and Planetary Science Letters 244, 186-200.

Zangerl, C., Evans, K.F., Eberhardt, E., Loew, S., 2008. Normal stiffness of fractures in granitic rock: A compilation of laboratory and in-situ experiments. International Journal of Rock Mechanics and Mining Sciences 45, 1500-1507.

Zimmerman, R.W., 2008. A Simple Model for Coupling between the Normal Stiffness and the Hydraulic Transmissivity of a Fracture, in: 42nd U.S. Rock Mechanics Symposium, San Francisco. 
Zimmerman, R.W., Bodvarsson, G.S., 1996. Hydraulic conductivity of rock fractures. Transport in Porous Media 23, 1-30.

Zimmerman, R.W., Chen, D.W., Cook, N.G.W., 1992. The effect of contact area on the permeability of fractures. Journal of Hydrology 139, 79-96.

Zimmerman, R.W., Chen, D.W., Long, J.C.S., Cook, N.G.W., 1990. Hydromechanical coupling between stress, stiffness, and hydraulic conductivity of rock joints and fractures, in: Barton, Stephansson (Eds.), Rock Joints. Balkema, Rotterdam, pp. 571-577.

Zoback, M.D., 2007. Reservior Geomechanics. Cambridge University Press. 